\title{
Long-term stroke prevention: We can do better
}

\author{
Michael D. Hill MD MSc
}

Cite as: CMAJ 2017 July 24;189:E952-3. doi: 10.1503/cmaj.170410

See related article at www.cmaj.ca/lookup/doi/10.1503/cmaj.161142

$\mathbf{T}$ reatments for acute ischemic stroke - including thrombolysis with alteplase and, more recently, endovascular thrombectomy - have received much attention. ${ }^{1-3}$ Efforts to find better treatments have focused mainly on early risks associated with stroke, particularly for patients with minor ischemic stroke or transient ischemic attack (which represent the mildest end of the ischemic stroke spectrum); many clinical trials are active in this area. Yet, the long-term risk of recurrent stroke lurks in the shadows.

Stroke is under-recognized as a major public health problem and incorrectly considered to be a disease that affects only older people. In North America and Western Europe, stroke is the third or fourth leading cause of death overall, but in the developing world is the first or second, depending on the country: globally, stroke is the second leading cause of death.

In Canada, despite recent capacity-building, there are still not nearly enough neurologists or stroke specialists to manage all patients with stroke. Patients in urban centres may be seen by a neurologist during the acute hospital admission, perhaps once or twice in follow-up, and then they are discharged to community follow-up. In rural settings, there is likely to be no stroke specialist at all. The long-term care of patients who have had a stroke falls to primary care physicians and internists. In this context, the findings of a linked study that examined the long-term risk of stroke are enlightening and shocking at the same time. ${ }^{4}$

Edwards and colleagues used administrative data to study Ontario patients who survived their initial ischemic stroke event, were discharged from the emergency department or from the hospital, and did not experience any further event for 90 days. Cases were matched with nonstroke controls on age, date of recent health system interaction, income quintile and area of residence. Outcomes were tracked for up to five years to examine death, recurrent stroke, myocardial infarction and admission to long-term care.

Stroke is an episodic condition that falls broadly into two categories: ischemic and hemorrhagic. Unlike acute coronary syndromes, which have a dominant underlying mechanism of coronary atherosclerotic disease, the possible causes of stroke are many. As a future coronary event depends upon atherosclerosis, so too the risk of future stroke depends upon the stroke mechanism.

\section{KEY POINTS}

- Ischemic stroke has many possible underlying causes.

- Specific treatments to prevent recurrent stroke depend on the underlying mechanism.

- In event-free survivors of stroke, recurrent stroke rates are high and can likely be reduced with attention to treatments aimed at influencing the stroke mechanism.

Edwards and colleagues found that cases with a high burden of hypertension, diabetes mellitus and prior stroke had twice the risk of the composite outcome of death, stroke and admission to long-term care or myocardial infarction compared with controls. Death was the most common outcome, followed by recurrent stroke. Patients who had experienced ischemic stroke were twice as likely to suffer recurrent stroke (all stroke - ischemic and hemorrhagic) as myocardial infarction. At five years, $7.8 \%$ of cases had experienced another stroke compared with $1.6 \%$ of controls, and $26 \%$ had died compared with $16 \%$ of controls. ${ }^{4}$ These findings underscore the importance of long-term risks for patients who appear to do well in the short term following stroke or transient ischemic attack.

Although this high mortality is likely explained by both the average population age of 72 and major comorbid illness (e.g., malignant disease and cognitive impairment or dementia), the major concern is recurrent stroke. Ischemic stroke is preventable and such high observed rates of recurrent stroke in the longer term suggest that there is opportunity to improve stroke prevention care. We can examine this opportunity by mechanistic subtype of ischemic stroke.

Ischemic stroke associated with atrial fibrillation can be prevented largely with anticoagulation. The introduction of and the ongoing gradual transition to use of direct oral anticoagulants in prevention should, in theory, reduce the burden of ischemic stroke among those with known nonvalvular atrial fibrillation. ${ }^{5-7}$ However, as many as $30 \%$ of patients with atrial fibrillation are first diagnosed when they present with a stroke. ${ }^{8}$ Atrial fibrillation is increasingly prevalent as age demographics change. Simple community-based screening for atrial fibrillation, such as the 
Program for the Identification of "Actionable" Atrial Fibrillation (www.cspin.ca/studies/piaaf/), may be a logical response to the problem of diagnosing asymptomatic atrial fibrillation before a stroke occurs. Further, anticoagulation for stroke prevention continues to have an incomplete penetrance in the at-risk population; not all patients with atrial fibrillation receive anticoagulant medication appropriately to prevent ischemic stroke. Ongoing trials among patients with embolic stroke but without evidence of atrial fibrillation or another cause (embolic stroke of undetermined source [ESUS] $)^{9}$ are testing direct oral anticoagulants compared with acetylsalicylic acid; if positive, these trials could revolutionize stroke prevention treatment by eliminating the need to identify silent or occult atrial fibrillation. Patients with stroke due to ESUS could simply be anticoagulated empirically.

Ischemic stroke due to large artery disease may be arteroembolic from extracranial carotid artery atherosclerosis or extracranial vertebral artery atherosclerosis, or it may be due to in situ thrombosis on a ruptured atherosclerotic plaque in an intracranial cerebral artery. A minority ( $10 \%$ or fewer) of ischemic stroke patients are suitable candidates for surgical or endovascular treatment of extracranial carotid disease. ${ }^{10}$ There is strong evidence that medical management is superior to angioplasty and stenting for intracranial atherosclerotic disease. ${ }^{11}$ Management therefore typically consists of medical treatment with antiplatelet agents, lipid-lowering agents and blood pressure control. However, it is the modifiable risk factors - smoking, exercise and diet - that continue to be the most challenging to address.

Ischemic stroke due to small vessel disease is prevented only with generic risk factor management. Optimal treatment of blood pressure ${ }^{12}$ and eschewing smoking are the definitive treatments. Although we suspect it is helpful, it is not known whether treatment of diabetes mellitus or using lipid-lowering therapy specifically prevents lacunar stroke. Other, less common causes of stroke may have specific preventive approaches, but their impact on overall recurrence rates will be proportionately small.

Edwards and colleagues highlight an unacceptably high rate of recurrent stroke among patients who survive their initial stroke and remain event free for 90 days. These stroke recurrence rates give us a benchmark upon which to improve. Stroke prevention is challenging because there are numerous possible causes to be addressed. However, there are proven treatments that work and are known to be underutilized. It is our imperative to use these treatments comprehensively and reduce recurrent stroke rates.

\section{References}

1. Hill MD, Buchan AM; Canadian Alteplase for Stroke Effectiveness Study (CASES) Investigators. Thrombolysis for acute ischemic stroke: results of the Canadian Alteplase for Stroke Effectiveness study. CMAJ 2005;172:1307-12.

2. Goyal M, Demchuk AM, Menon BK, et al. Randomized assessment of rapid endovascular treatment of ischemic stroke. N Engl J Med 2015;372:1019-30.

3. Lambrinos A, Schaink AK, Dhalla I, et al. Mechanical thrombectomy in acute ischemic stroke: a systematic review. Can J Neurol Sci 2016;43:455-60.

4. Edwards JD, Kapral MK, Fang J, et al. Long-term morbidity and mortality in patients without early complications after stroke or transient ischemic attack. CMAJ 2017;189: E954-61.

5. Connolly SJ, Ezekowitz MD, Yusuf S, et al. Dabigatran versus warfarin in patients with atrial fibrillation. N Engl J Med 2009;361:1139-51.

6. Patel MR, Mahaffey KW, Garg J, et al. Rivaroxaban versus warfarin in nonvalvular atrial fibrillation. N Engl J Med 2011;365:883-91.

7. Connolly SJ, Eikelboom J, Joyner C, et al. Apixaban in patients with atrial fibrillation. N Engl J Med 2011;364:806-17.

8. Yu AY, Malo S, Wilton S, et al. Anticoagulation and population risk of stroke and death in incident atrial fibrillation: a population-based cohort study. CMAJ Open 2016;4:E1-6.

9. Hart RG, Diener HC, Coutts SB, et al. Embolic strokes of undetermined source: the case for a new clinical construct. Lancet Neurol 2014;13:429-38.

10. Rothwell PM, Eliasziw M, Gutnikov SA, et al. Endarterectomy for symptomatic carotid stenosis in relation to clinical subgroups and timing of surgery. Lancet 2004; 363:915-24.

11. Chimowitz MI, Lynn MJ, Derdeyn CP, et al. Stenting versus aggressive medical therapy for intracranial arterial stenosis. N Engl J Med 2011;365:993-1003.

12. SPS3 Study Group, Benavente OR, Coffey CS, Conwit R, et al. Blood-pressure targets in patients with recent lacunar stroke: The SPS3 randomised trial [published erratum in Lancet 2013;382:506]. Lancet 2013;382:507-15.
Competing interests: Michael Hill reports personal fees from Merck, nonfinancial support from Hoffmann-La Roche Canada Ltd., and grants from Covidien (Medtronic Minimally Invasive Therapies), Boehringer Ingelheim, Medtronic, NoNO Inc. and Stryker Inc., outside the submitted work. In addition, he has a patent pending for systems and methods for assisting in decision-making and triaging for acute stroke patients to the US Patent Office, number 62/086,077. He owns stock in Calgary Scientific Inc., a company that focuses on medical imaging software; is a director of the Canadian Neurological Sciences Federation, a not-for-profit group; and has received grant support from Alberta Innovates: Health Solutions, the Canadian Institutes of Health Research, the Heart and Stroke Foundation of Canada and the National Institutes of Neurological Disorders and Stroke.

Affiliations: Department of Clinical Neurosciences, University of Calgary, and Hotchkiss Brain Institute, Calgary, Alta.

This article was solicited and has not been peer reviewed.

Correspondence to: Michael Hill, michael.hill@ucalgary.ca 